\title{
Um método para implementação de Comitê Ágil de Governança de TI para inovação de processos e ferramentas
}

\author{
Adriano Galindo Leal, Matheus Jacon Pereira, Leandro Avanço, Igor Cunha \\ Teixeira, Alessandro Santiago dos Santos, Rodrigo Neves Ribeiro
}

Centro de Informação, Automação e Mobilidade - Instituto de Pesquisas Tecnológicas, Av. Prof. Almeida Prado, 532 - Butantã, São Paulo - SP, 05508-901 - Brasil

\{leal, mjacon, lavanco, igort, alesan, rodrigoneves\}@ipt.br

\begin{abstract}
The present case study describes a method of implementing IT Governance based on the formation of an Agile Committee to restructure inadequate bureaucratic processes, aiming to meet the demands for improving the efficiency of the use of governmental resources by public and mixed economy companies. This method requires the formation of a Governance Committee with the necessary authority and freedom to assess and implement changes in existing processes and systems. After 25 months of application at a Public Institution linked to the Government of the State of São Paulo, the equivalent COBIT 5.0 processes APO01, APO05, APO13, BAI01, BAI04, BAI06, BAI08, BAI09, DSS01, DSS02 and DSS05 of the Support Management pillar evolved from Level 0 of capacity (Incomplete), mostly to Level 1, Performed. Only the processes DSSO1 and DSSO2 reached Level 2 (Managed).
\end{abstract}

Resumo. O presente estudo de caso descreve um método de Implementação de Governança de TI baseado na formação de um Comitê Ágil para reestruturar processos burocráticos inadequados, visando atender às demandas de melhoria na eficiência do uso de recursos públicos por empresas e sociedades de economia mista. Esse método demanda a formação de um Comitê de Governança com a necessária autoridade e liberdade para avaliar e implementar mudanças nos processos e sistemas existentes. Depois de 25 meses de sua aplicação em uma Instituição Pública ligada ao Governo do Estado de São Paulo, os processos equivalentes ao COBIT 5.0 do pilar de Gestão de Suporte (APO01, APO05, APO13, BAI01, BAI04, BAI06, BAI08, BAI09, DSS01, DSSO2 and DSS05) evoluíram do Nivel 0 de capacidade (Incompleto), em sua maioria para o Nivel 1, Realizado. Apenas os processos DSSO1 e DSS02 atingiram o Nivel 2 (Controlado).

\section{Introdução}

A Lei $\mathrm{N}^{0} 13.303$ submete as empresas públicas e sociedades de economia mista às regras de governança, que engloba obrigatoriamente a TI, é uma das respostas para uma crescente demanda da sociedade por melhorias na eficiência da gestão e controle em órgãos públicos, sejam eles empresas estatais, fundações, entidades de administração indireta, bem como autarquias, institutos e universidades (Brasil, 2016). Além disso, existe a necessidade de se adaptar à uma série de iniciativas de transparência dos dados públicos e aspectos de segurança da informação vêm sendo exigidas por força de lei, sendo que devemos destacar a Lei Geral de Proteção de Dados Pessoais, Lei no 13.709, que regula as atividades de tratamento de dados pessoais (Brasil, 2018). 
O ambiente de Tecnologia da Informação e Comunicação (TIC) está em constante evolução normativa e tecnológica. Empresas públicas e sociedades de economia mista são construções sociais coletivas complexas regrada pela legislação vigente e, frequentemente, submetidas a ciclos de três anos de adaptações e ajustes profundos, tanto em sua estrutura organizacional, como em sua missão, de forma a atenderem projetos prioritários, buscando o bem comum, do Governo Vigente.

A experiência no presente estudo de caso validou a percepção que os Gestores Públicos Brasileiros vêm paulatinamente, em face da promulgação das leis previamente mencionadas, se conscientizando que a Governança em TI (GTI) é um pilar fundamental para essa adaptação ao novo ambiente e necessita adaptar processos e encontrar ferramentas que possam suprir tais demandas por serviços qualificados e eficazes. Uma vez estabelecido que o Gestor irá devotar os recursos necessários para suportar essa atividade, existe uma pletora de decisões de alta complexidade e impactos de curto, médio e longo prazo que devem ser ponderados para que se evite que recursos humanos e financeiros sejam desperdiçados em aquisições tecnológicas mal realizadas (Sena, 2018). Este complexo processo de adoção e gerenciamento das TIC's se torna um desafio ainda maior dentro da administração pública que têm problemas como recursos orçamentários insuficientes e falta de comprometimento organizacional com a Governança, além de servidores em número insuficiente e, em sua maioria, desatualizados com a tecnologia e boas práticas vigentes no mercado e na academia.

O modelo de Governança de TI deve incentivar a criação de uma cultura corporativa de práticas de avaliação e monitoração dos processos, assegurando, um nível aceitável de risco, uma eficiente utilização dos recursos que apoiam os processos e o ambiente estratégico com os objetivos institucionais (Putz, Rasoto, \& Ishikawa, 2017).

O objetivo deste trabalho é apresentar um método de Implementação de Governança de TI em Empresas Públicas que realizam atendimento à população, baseado nos principais frameworks (ITIL, COBIT e PMBOK). Ao final, serão apresentados os resultados de uma aplicação deste método.

Este trabalho está organizado da seguinte forma: a seção 2 apresenta a descrição do método. A seção 4 analisa os resultados da aplicação desse método e, por fim, as considerações finais encerram a seção 5.

\section{Método de Implementação de Comitê de Governança de TI}

O método proposto neste artigo é baseado na experiência prática da implementação, em órgão governamental, das melhores práticas dos principais frameworks de governança de TI, a saber, ITIL, COBIT, COSO, BSC e PMBOK. Este método apresenta alternativas realistas para atender as necessidades de modernização dos recursos de TIC e eficiência no gasto dos recursos financeiros de empresas da administração pública, principalmente daquelas que prestam serviços diretamente ao público.

O método consiste na formação de um Comitê de Governança dotado de autoridade e liberdade para avaliar e implementar mudanças nos processos e sistemas existentes. $\mathrm{O}$ cuidado basilar é incluir toda a cadeia hierárquica até a Presidência da Instituição, não se restringindo à participação da Alta Gerência da TI, pois isso garante que haverá apoio institucional para a elaboração e manutenção das políticas da Governança de TI em consonância com a Legislação Vigente. Em complemento, o core do Comitê também é 
formado por profissionais independentes dos processos existentes e dotados de sólidos conhecimentos em diversas áreas de TIC, partindo de infraestrutura de TI, passando por desenvolvimento de software, administradores de banco de dados e experiência do usuário, sem deixar de fora a comprovada experiência em Governança de TI. Ainda, faz parte desse comitê um representante por processo estudado/afetado de forma a completar de forma reduzida o conjunto de stakeholders.

Seguindo uma tradicional recomendação SCRUM, oriunda de (Schwaber \& Sutherland, 2017), que recomenda que "o tamanho ideal do Time de Desenvolvimento é pequeno o suficiente para se manter ágil e grande o suficiente para completar uma parcela significativa do trabalho dentro dos limites da Sprint", deliberamos que a qualquer tempo, que o total de profissionais participantes não ultrapasse 10 (dez) pessoas, devido ao risco de perda de foco, ansiedade generalizada, indecisão e paralisia. Da mesma forma, não trabalhar com um total menor que 5 (cinco) pessoas para evitar situações de despotismo, corporativismo e limitação da diversidade de conhecimento do pool dos profissionais envolvidos.

Para atender às demandas da organização, o Comitê de Governança inicialmente faz um mapeamento do Portfólio de Serviços e escolhe o processo mais importante, o qual não possua indicadores-chave de desempenho (em inglês Key Performance Indicator - KPI), levando em consideração o impacto na cultura organizacional, a dependência de pessoas e o nível de serviço esperado. O desenvolvimento de novos KPIs serão usados para acompanhar, executar e monitorar as decisões referentes aos planos de ação propostos para TI para alinhamento com a estratégia de negócios. Consequentemente, mede-se a eficiência e a Governança, permitindo ainda, auditar o SLA (Nível de Serviço Acordado, do inglês Service Level Agreement).

Uma vez que o Comitê de Governança nomeie o processo que deverá ser melhorado, lembrando-se de selecionar representantes dos setores afetados enquanto mantenha o limite total de 10 (dez) pessoas, deverá se proceder a Etapa Pesquisa (Figura 1) aonde irá se fazer um redesenho idealístico do processo levando em conta a digitalização da sociedade e tecnologias atuais. A partir desse redesenho idealístico, procede-se em avaliar tanto a viabilidade de recursos disponíveis quanto de ferramentas computacionais disponíveis de acordo com suas funcionalidades, sejam elas proprietárias ou de código aberto.

Deve-se ressaltar que a atividade de redesenho de processo é iterativa e permeia todas as etapas ilustradas na Figura 1, uma vez que serão necessários inúmeros ajustes até que a versão final do processo seja registrada junto ao Setor de Controle da Qualidade. Devese frisar o aspecto que o primeiro redesenho seja idealístico, mas levando-se em conta os reais limitantes do escopo da Organização e da Legislação Vigente. de a versão final contenha elementos de real inovação que dê um salto qualitativo na qualidade, transparência e fluidez dos processos e não a simples automação de uma burocracia que não encontra mais lugar no e-Gov das cidades inteligentes (Smart Cities).

O Comitê de Governança seleciona e avalia as opções das ferramentas computacionais que irão atender as demandas da organização. Para isso utiliza uma sequência básica de implementação de processos de governança, a qual consiste nas etapas da Figura 1, que serão detalhados nos subitens desta seção. 


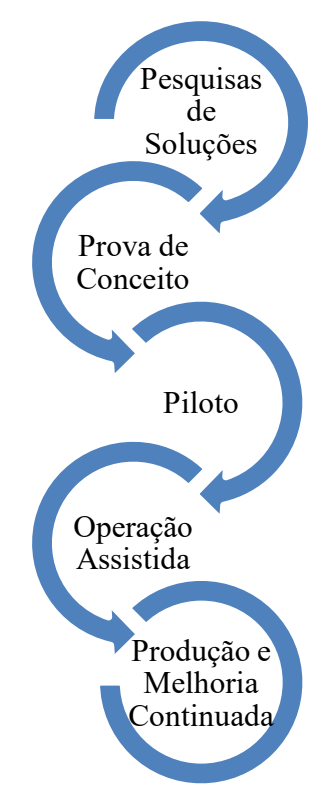

Figura 1 - Etapas para implementação de ferramentas de governança

\subsection{Pesquisas de Soluções}

A pesquisa de soluções é iniciada por meio de reuniões com a equipe da empresa para discutir os objetivos de médio e longo prazo e, tendo esses como premissas, avaliar os requisitos técnicos e de negócio para atingi-los. Entre os requisitos, é valido citar: recursos necessários (técnicos, humanos e negócios), funcionalidades esperadas, perfis de uso, interfaces de usuário e, integração aos demais sistemas em operação.

Nesta etapa é realizada uma pesquisa de ferramentas de software existentes no mercado, sejam elas proprietárias ou livres (Open Source). Cada uma dessas ferramentas pesquisadas é avaliada utilizando critérios técnicos estabelecidos nas reuniões. Aonde, obrigatoriamente, deve-se levar em conta a curva de aprendizado, custo de propriedade estimado, disponibilidade do código, equipe de suporte para Implementação dentro da organização, bem como o tempo para preparação e execução do Edital de Compra Governamental. Ao término desta etapa, um relatório técnico é elaborado contendo as informações detalhadas das ferramentas e o resultado da avaliação.

\subsection{Prova de Conceito}

O relatório elaborado na etapa anterior pode escolher uma ou mais ferramentas para realização de uma demonstração à área de negócio interessada. Cada demonstração é denominada Prova de Conceito (POC) e permite que a área de negócio e área técnica avalie a ferramenta com base nos critérios pré-definidos. A avaliação e escolha da ferramenta possibilita que o negócio selecione a solução mais apropriada a suas necessidades de médio e longo prazo, a qual seguirá para fase de Piloto.

\subsection{Piloto}

A etapa de Piloto consiste na realização da implementação da solução escolhida na etapa a anterior. Nesta, um cuidadoso planejamento da transição deverá ser realizado, o processo anteriormente utilizado pode ou não começar a ser abandonado, uma vez que, em geral, as equipes são muito reduzidas e tal fato pode ser impeditivo para a operação 
em paralelo. O piloto permite a avaliação de viabilidade e impacto efetivos do novo processo e da atividade de TIC necessária para a sua manutenção.

Nesta etapa, os novos procedimentos e atividades são refinados buscando aprimorar o novo processo e sua interação com o ecossistema de TIC remanescente. Os procedimentos e os processos são avaliados criticamente visando sua efetiva implementação. Além disso, os testes unitários e de integração com demais soluções são realizados nesta etapa. Ao final desta etapa, é realizada a validação e homologação do sistema, bem como de cada nova funcionalidade.

\subsection{Operação Assistida}

A etapa de Operação Assistida consiste na Implementação da solução no ambiente de produção, bem como o acompanhamento da operação da solução após a Implementação e a transferência de conhecimento tanto para a equipe que ficará responsável pelo processo de sustentação e treinamento operacional da solução de TIC, quanto para a que utilizará a solução.

É importante destacar que, o Comitê de Governança contém os profisssionais que irão coordenar e realizar as atividades até o momento da transferência de conhecimento, após isso, deve-se repassar essas atividades para as equipes tradicionais. Desta forma, o Comitê volta a ter disponibilidade para focar na reestruturação de outros processos da Instituição que estejam apresentando ineficiência ou que necessitem se modernizar frente à Digitalização da Sociedade.

A Implementação da solução é realizada instalando os elementos computacionais necessários ao funcionamento da solução, configuração da solução de acordo com as necessidades do negócio e integração da solução com demais sistemas da Instituição.

O acompanhamento da operação da solução é realizado assim que a Implementação da solução esteja concluída. A infraestrutura e os serviços de TIC são monitorados para garantir o devido funcionamento da solução. A utilização da solução pela equipe de negócio é acompanhada para identificar qualquer anormalidade na solução e corrigir o mais rápido possível.

A transferência de conhecimento é realizada por meio de treinamentos técnicos para os responsáveis pela operação e sustentação da solução. Os treinamentos operacionais são realizados para a equipe de negócio que utiliza a solução. Os treinamentos de governança de TIC são realizados para todos os envolvidos no processo, sendo que os gestores operacionais e técnicos são treinados para garantir que não ocorram desvios de processo no início de operação e ao longo de seu ciclo de vida. Os demais membros das equipes são treinados para executar o processo de acordo com o acordado na etapa de Pesquisas de Solução.

\subsection{Produção e Melhoria Continuada}

A etapa Produção e Melhoria Continuada garante que os serviços estejam alinhados com as necessidades atuais do negócio. Nesta, as mudanças demandadas pelo negócio devem ser identificadas por meio de um processo de melhoria contínua.

A implementação das melhorias no serviço deve ser realizada pela equipe técnica de forma garantir a evolução do negócio. 


\section{Resultados}

O método proposto neste artigo foi aplicado no projeto de Implementação de GTI em uma Instituição Pública do Governo do Estado de São Paulo. Esse projeto foi estruturado em 4 (quatro) pilares, ilustrados na Figura 2:

- Modelo de Governança - Esse pilar teve como objetivo o desenvolvimento e a implementação de uma metodologia de Governança de TIC;

- Gestão de Suporte - Esse pilar teve como objetivo a estruturação, implementação e otimização de um Service Desk, como ponto de contato para as demandas de serviços de TIC;

- Gestão de Operação - Esse pilar teve como objetivo implementar um Centro de Operação de Rede (Network Operation Center - NOC) para coordenar e executar as atividades e procedimentos operacionais necessários para entregar os serviços de TI internamente e terceirizados;

- Segurança da Informação - Esse pilar teve como objetivo implementar os processos de relacionados à segurança da informação para definir, operar e monitorar um Sistema de Gestão de Segurança da Informação (SGSI) buscando proteger as informações da empresa e manter o nível de risco de segurança da informação aceitável, de acordo com a política de segurança.
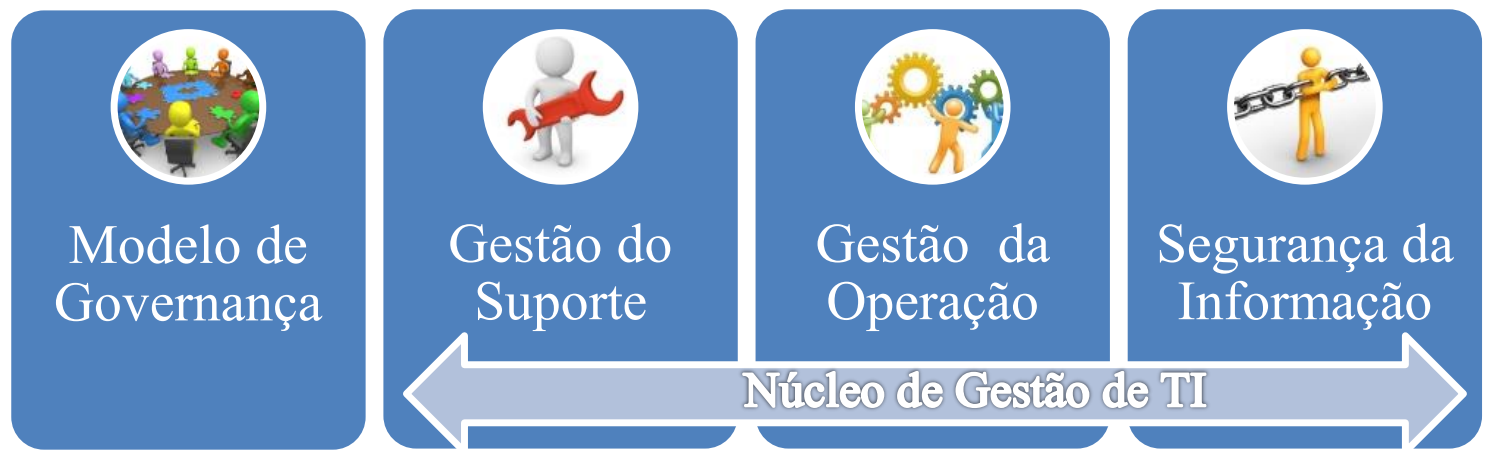

Figura 2 - Pilares de Governança de TIC

As atividades executadas ao longo do projeto foram alinhadas de forma ágil com o cliente para atender suas necessidades sempre de acordo com o método proposto. $\mathrm{O}$ pilar Gestão do Suporte foi elencado pelo cliente como item estratégico, portanto, foi o primeiro dos pilares a ser abordado no projeto. Obviamente, o Pilar do Modelo de Governança teve uma atividade constante durante todo o projeto, embora a prioridade fosse a Gestão do Suporte pois era o que mais necessitava de reformulação.

O objetivo principal do pilar Gestão do Suporte foi estruturar, implementar e otimizar um Service Desk, permitindo que opere como ponto único de contato para as atender as demandas de serviços de TI abrangendo as seguintes atividades:

- Atendimento a incidentes, problemas e dúvidas;

- Atendimento a solicitações de mudança;

- Controle de licenças de software;

- Gerenciamento de nível de serviço;

- Gerenciamento de configuração. 
Seguindo o método, na etapa Pesquisas de Soluções, foi realizado um levantamento inicial do Service Desk existente e constatou-se o uso de um sistema de registro de chamados rudimentar. O sistema anterior não oferecia controle de acesso, consulta ao andamento dos chamados, histórico dos chamados abertos, controle de prioridades para os chamados e nenhuma opção de relatório ou dashboard gerencial. Além disso, o processo anteriormente adotado permitia que os usuários acionassem o suporte de TIC pelo sistema, por e-mail, por telefone e presencialmente, sem que a obrigatoriedade de registro dos chamados. Essa característica impedia o pleno rastreamento dos chamados e atividades do setor.

As etapas seguintes (Prova de Conceito e Piloto) resultaram no redesenho dos processos constituintes do pilar de Gestão de Suporte. Para auxiliar o processo redesenhado, durante essas etapas, foi realizado a Implementação da ferramenta Open Source GLPI (Teclib, 2015). Mesmo assim, o processo foi refinado durante a Etapa Operação Assistida para aperfeiçoar o uso da ferramenta. Nesta etapa, as áreas usuárias foram treinadas para adequação ao novo processo e para o uso da ferramenta. A equipe de TI foi treinada no novo processo buscando a aderência e para auxiliar os usuários a se adequar a mudança no processo. $\mathrm{O}$ treinamento no uso da ferramenta foi importante para conscientizar a equipe sobre novos recursos e sua importância para os indicadores gerenciais.

Ao concluir a Etapa Produção e Melhoria Continuada, o pilar de Gestão de Suporte obteve total rastreabilidade e um índice de $80 \%$ dos chamados fechados em até de 1 (um) dia. A ferramenta adotada foi expandida para outras áreas, além do suporte de TI. Entre os principais entregáveis para o Pilar de Gestão de Suporte, cita-se:

- Visualização do andamento e histórico dos chamados pelo próprio usuário;

- Informações Gerenciais por meio de Dashboard;

- Funcionalidades para o gerenciamento de problemas, mudanças e contratos.

Tabela 1 - Nível de capacidade dos processos da Gestão de Suporte

\begin{tabular}{|l|l|}
\hline Processos do COBIT 5 & Avaliação \\
\hline APO01 Gerenciar o Framework de Gestão de TI & Nível 1 - Processo Realizado \\
\hline APO05 Gerenciar o Portfólio & Nível 1 - Processo Realizado \\
\hline APO13 Gerenciar a Segurança & Nível 1 - Processo Realizado \\
\hline BAI01 Gerenciar Programas e Projetos & Nível 1 - Processo Realizado \\
\hline BAI04 Gerenciar a Disponibilidade e Capacidade & Nível 1 - Processo Realizado \\
\hline BAI06 Gerenciar Mudanças & Nível 1 - Processo Realizado \\
\hline BAI08 Gerenciar o Conhecimento & Nível 1 - Processo Realizado \\
\hline BAI09 Gerenciar os Ativos & Nível 1 - Processo Realizado \\
\hline DSS01 Gerenciar as Operações & Nível 2 - Processo Gerenciado \\
\hline DSS02 Gerenciar Requisições de Serviço e Incidentes & Nível 2 - Processo Gerenciado \\
\hline DSS05 Gerenciar Serviços de Segurança & Nível 1 - Processo Realizado \\
\hline
\end{tabular}

Um aspecto que é importante destacar é que o pilar Gestão de Suporte, denominação utilizada pelo Órgão estudado, pode ser dividido nos processos COBIT 5 exibidos na Tabela 1, embora não os empregue exatamente essa nomenclatura. No início do projeto, quase a totalidade dos processos era executada de forma ad-hoc (Nível 0 - Incompleto).

Após 25 meses de aplicação do método proposto, os autores realizaram uma nova avaliação do nível de capacidade de cada um dos processos da Gestão de Suporte sob a 
ótica dos processos do Cobit 5.0 COBIT (ISACA, 2012). Ambos os resultados foram submetidos a um consultor especialista em ITIL e COBIT, que teve acesso à empresa durante o projeto para questões de treinamento, mas não se envolveu nos trabalhos do Comitê. Desta forma, foram minimizados, dentro do plausível, eventuais vieses dos autores. Outros processos do Cobit 5.0 não foram analisados por não fazerem parte do escopo do departamento contratante do projeto, bem como outras condições de contorno decorrentes de normativas da empresa.

\section{Considerações Finais}

O método proposto tem como ponto fulcral a formação de um Comitê de Governança, usando filosofia SCRUM, com a necessária autoridade e liberdade para avaliar e implementar mudanças nos processos e sistemas existentes na organização.

Embora ainda tenha sido importante, para o sucesso deste tipo de projeto, o empenho da Presidência do Órgão e a Alta Hierarquia no estabelecimento da obrigatoriedade em se seguir os processos redesenhados, as Leis Federais $\mathrm{N}^{0} 13.303 / 2016$ e Lei $\mathrm{n}^{\mathrm{o}}$ 13.709/2018, propriamente regulamentadas de forma a pormenorizar as disposições gerais e abstratas da lei, viabilizando sua aplicação em casos específicos, trarão grande impulso e maturidade à Governança de TI no ambiente das Instituições e Órgãos Públicos Brasileiros.

A aplicação do método foi realizada em um projeto de Implementação de Governança de TI em uma Instituição Pública ligada ao Governo do Estado de São Paulo. Após 25 meses, a maioria dos processos constituintes do pilar de Gestão de Suporte, equivalentes ao COBIT 5.0, evoluíram do Nível 0 de capacidade (Processo Incompleto), em sua maioria para o Nível 1, Processo Realizado e apenas os processos DSS01 e DSS02 atingiram o Nível 2 (Processo Controlado).

\section{Referências}

Brasil. (30 de junho de 2016). Lei $n^{o} 13.303$ - Estatuto Jurídico da Empresa Pública e da Sociedade de Economia Mista. Acesso em 13 de 02 de 2020, disponível em http://www.planalto.gov.br/ccivil_03/_ato2015-2018/2016/lei/113303.htm

Brasil. (2018). Lei $n^{o} 13.709$ - Lei Geral de Proteção de Dados Pessoais (LGPD). Acesso em 13 de 02 de 2020, disponível em http://www.planalto.gov.br/ccivil_03/_ato2015-2018/2018/lei/L13709.htm

ISACA. (2012). COBIT 5. ISACA.

Putz, R., Rasoto, V., \& Ishikawa, E. (2017). Brazilian federal universities information technology governance: An analysis of the strategic alignment dimension. 12th Iberian Conference on Information Systems and Technologies (CISTI). IEEE.

Schwaber, K., \& Sutherland, J. (2017). The Scrum Guide: The Definitive The Rules of the Game. Scrum.Org and ScrumInc, 19. doi:10.1053/j.jrn.2009.08.012

Sena, I. (2018). Governança de TI em Órgãos Públicos: Um Mapeamento Sistemático da Literatura.

Teclib. (2015). GLPI. Acesso em 13 de 02 de 2020, disponível em https://glpiproject.org/pt-br/ 